\title{
INTERACCIÓN GENOTIPO AMBIENTE Y POTENCIAL PRODUCTIVO DE 25 VARIEDADES DE MAÍz AMILÁCEO EN LA PROVINCIA DE TAYACAJA, PERÚ
}

\author{
Pedro J. García-Mendoza ${ }^{1}$, Iris B. Pérez-Almeida ${ }^{2}$, Gino P. Prieto-Rosales ${ }^{1}$, Darío E. Medina- \\ Castro $^{3}$, Damián Manayay Sánchez ${ }^{4}$, Carlos A. Marín-Rodríguez ${ }^{5}$, Juan C. Ricse Nestares ${ }^{6}$, \\ Ronald Ortecho Llanos ${ }^{1}$ y Alicia E. Medina-Hoyos ${ }^{7}$
}

\section{RESUMEN}

La interacción genotipo por ambiente (IGA) representa el principal obstáculo en la selección de los genotipos promisorios para diversos ambientes. El objetivo de esta investigación fue evaluar la IGA y el potencial productivo de 25 variedades de maíz amiláceo, utilizando el modelo de efectos principales aditivos e interacción multiplicativa (AMMI). Se utilizó la información generada en cinco ensayos establecidos en diferentes localidades de la provincia de Tayacaja, Huancavelica, Perú, en el ciclo del cultivo 2018-2019, bajo un diseño alfa látice $5 \times 5$, con tres repeticiones y unidades experimentales de dos hileras de $4 \mathrm{~m}$ de longitud, con arreglos espaciales de $0,80 \mathrm{~m}$ entre hileras y $0,20 \mathrm{~m}$ entre puntos de siembra. El potencial productivo de los cultivares se midió a través del rendimiento de grano, ajustado a $15 \%$ de humedad. Definida la IGA, se realizó el análisis multivariado, para obtener los valores singulares de los primeros términos significativos del modelo para genotipos y ambientes. La IGA resultó altamente significativa y explicó alrededor del $14 \%$ de la variación fenotípica del rendimiento, mostrando algunas variedades con adaptación específica y otras con amplia adaptación a los ambientes de prueba. El modelo AMMI explicó alrededor del $96 \%$ de la variación debida a la IGA, observándose que sólo los dos primeros ejes concentraron cerca del $85 \%$ de dicha variación. Las variedades locales Chullpi-Q, Carhuay-P, Astilla Blanca-Cusqueado, Astilla Blanca-DH, Cusqueado-P y Astilla Blanca-Astilla Amarilla mostraron el mejor potencial productivo, pero sólo las tres primeras fueron estables a través de los ambientes de evaluación.

Palabras clave adicionales: Adaptabilidad, modelo AMMI, rendimiento, Zea mays

\begin{abstract}
Genotype by environment interaction and productive potential of 25 starch maize assessed at the Tayacaja Province, Peru The genotype by environment interaction (GEI) represents a main difficulty in the selection of promising genotypes for different environments. The objective of this research was to evaluate the GEI and the productive potential of 25 starch maize varieties, using the additive main effects and multiplicative interaction (AMMI) model. The information was collected from five trials in different locations of the Tayacaja province, Peru, during the 2018-2019 season, under an alfa lattice 5x5 experimental design with three replications and experimental units of two rows $4 \mathrm{~m}$ long, $0.80 \mathrm{~m}$ between rows and $0.20 \mathrm{~m}$ between seeding points. The cultivar productive potential was measured through the grain yield, adjusted to $15 \%$ moisture. Once the GEI was defined, the multivariate analysis was carried out to obtain the singular values of the first significant model terms for genotypes and environments. The GEI was highly significant and explained around $14 \%$ of the yield phenotypic variation, discriminating some varieties with specific adaptation, while others showed broad adaptation to the environments. The AMMI model explained about $96 \%$ of the variation due to the GEI, showing that only the first two axes concentrated about $85 \%$ of that variation. The local varieties Chullpi-Q, Carhuay-P, Astilla Blanca-Cusqueado, Astilla Blanca-DH, Cusqueado-P and Astilla Blanca-Astilla Amarilla showed the best productive potential, but only the first three remained stable through the evaluation environments.
\end{abstract}

Additional keywords: Adaptability, AMMI model, yield, Zea mays

Recibido: Junio 22, 2020

Aceptado: Enero 13, 2021

${ }^{1}$ Facultad de Ingeniería, Universidad Nacional Autónoma de Tayacaja, "Daniel Hernández Morillo" (UNAT), Pampas, Tayacaja, Perú. e-mail: pedrogarcia@ unat.edu.pe (autor de correspondencia); ronaldortecho@unat.edu.pe

${ }^{2}$ Centro de Estudios Desarrollo Sostenible, Universidad Ecotec, Samborondón, Ecuador. e-mail: iperez@ecotec.edu.ec

${ }^{3}$ Facultad de Biología, Universidad Nacional de Trujillo, Trujillo, Perú. e-mail: darikmedina_06@ hotmail.com

${ }^{4}$ Dpto. Agroindustria, Fac. Ingeniería, Universidad Nacional del Santa, Ancash, Perú. e-mail: dmanayay@ gmail.com

${ }^{5}$ Unidad Biometría y Estadística, Inst. Nac. Invest. Agríc (INIA). Maracay, Venezuela. e-mail: carlmarinr@gmail.com

${ }^{6}$ Proyecto maíz de la UNAT, Pampas, Tayacaja, Huancavelica, Perú. e-mail: nestares1308@ outlook.es

${ }^{7}$ Programa Nacional de Maíz, Inst. Nac. Innov. Agraria (INIA), Cajamarca, Perú. e-mail: aemedinah2@ hotmail.com 


\section{INTRODUCCIÓN}

La interacción genotipo ambiente (IGA), considerada un fenómeno universal cuando se prueban diferentes genotipos en ambientes contrastantes, representa el factor que determina el mayor porcentaje de inversión en los programas de mejoramiento genético, debido a la necesidad de evaluar una gran cantidad de genotipos en el mayor número de ambientes posibles (Dudley, 1997; Acevedo et al., 2019). Se trata de un fenómeno que dificulta la selección de los genotipos superiores, razón por la cual también representa uno de los mayores retos que enfrentan agrónomos y mejoradores de plantas, para recomendar los genotipos más convenientes para los diferentes ambientes de interés (Souza et al., 2009; Mudada et al., 2017).

Cuando se tiene un conjunto de variedades que pueden ser cultivadas por pequeños agricultores ubicados en regiones con marcadas diferencias agroecológicas, previo a recomendarles la variedad ideal para las diferentes regiones de producción, es esencial conocer la contribución del efecto de la IGA sobre la prospectiva variedad. Esto significa que cuando un grupo de genotipos son probados en ambientes contrastantes, se podrían identificar algunos de ellos con amplia adaptación a los diversos ambientes de prueba, mientras que otros mostrarían adaptación a condiciones específicas (Chimonyo et al., 2014).

Un análisis de varianza (ANOVA) combinado puede utilizarse para cuantificar el efecto de la IGA y describir los efectos principales de genotipos y ambientes. No obstante, el ANOVA, por sí solo, no puede explicar la totalidad del efecto de la IGA, de tal forma que se puedan identificar los genotipos con amplia adaptación o con adaptación específica (Admassu et al., 2008). Para explicar la interacción de los efectos principales más allá del ANOVA, deben emplearse otros modelos estadísticos, tales como el modelo de efectos principales aditivos e interacción multiplicativa AMMI, propuesto por Zobel et al. (1988). La metodología de análisis y los principios estadísticos y científicos que sustentan el método AMMI han sido bien detallados en trabajos previos (Gauch y Zobel, 1996; Crossa et al., 2000; Yan et al., 2007); así como la interpretación de los efectos que representan los valores de los primeros componentes principales respecto a la IGA (García et al., 2020).

En el Perú han sido pocos los trabajos de investigación referidos al estudio de la IGA, y de los publicados a la fecha en el cultivo de maíz (Chura y Huanuqueño, 2015; López et al., 2019) ninguno se ha realizado en la región de la sierra. De estos estudios, sólo el segundo ha utilizado la metodología empleada en esta investigación, lo cual se presume que es debido al hecho que es necesario evaluar un gran número de genotipos en diversos ambientes, acción que requiere no sólo la disponibilidad de logística y recursos económicos apropiados, sino también de contar con un equipo técnico capacitado y con disposición para desarrollar los experimentos en condiciones de ambientes que ameritan un esfuerzo físico adicional. En el Perú, el mayor énfasis se ha dado al desarrollo de cultivares de maíz para la región costera, posiblemente, debido a que representa la mayor área sembrada con ese cultivo y consecuentemente de mayor interés económico para las empresas de semillas que operan en el país.

La Universidad Nacional Autónoma de Tayacaja, "Daniel Hernández Morillo" (UNAT, 2018), viene desarrollando un proyecto de investigación agrícola en maíz, denominado "Selección de cultivares y conservación de la identidad genética de variedades de maíz (Zea mays L.) amiláceo en la provincia de Tayacaja”, cuyo principal objetivo es seleccionar y desarrollar variedades de maíz con elevado potencial genético de producción y calidad culinaria e industrial, que contribuyan a mejorar la rentabilidad de toda la cadena agro productiva vinculada al cultivo de maíz amiláceo en la Provincia de Tayacaja.

Este proyecto contempló la evaluación de un grupo de variedades de este cultivo recolectadas en diferentes condiciones de ambiente de la región de influencia, representando una oportunidad para obtener información nueva y relevante para orientar a los agricultores en la selección de las mejores variedades para las diferentes áreas agroecológicas. De esta forma, el objetivo principal de esta investigación fue evaluar la interacción genotipo ambiente y el potencial productivo de 25 variedades de maíz amiláceo en diferentes ambientes de la provincia, en el ciclo del cultivo 2018-2019, utilizando el modelo AMMI, a fin de facilitar la selección de los materiales más aptos para los 
diferentes ambientes productores de maíz amiláceo de la provincia de Tayacaja.

\section{MATERIALES Y MÉTODOS}

En los experimentos se utilizaron 19 variedades experimentales de maíz amiláceo recolectadas en la provincia de Tayacaja, Huancavelica, Perú, más 6 variedades mejoradas de maíz amiláceo proporcionadas por el Instituto Nacional de Innovación Agraria (INIA) Cajamarca (Cuadro 1).

Los ensayos fueron establecidos en 5 localidades, distribuidas en diferentes distritos productores de maíz amiláceo de la provincia (Cuadro 2). Se usó un diseño experimental de bloques incompletos, Alfa Látice $5 \times 5$, con tres repeticiones; la unidad experimental estuvo compuesta por dos hileras de $4 \mathrm{~m}$ de longitud, utilizando arreglos espaciales de $0,80 \mathrm{~m}$ de separación entre hileras y $0,20 \mathrm{~m}$ entre puntos de siembra; los experimentos fueron diseñados para colocar dos semillas por punto de siembra (84 semillas por parcela). Posteriormente, se realizó un raleo transcurridos 30 días después de la siembra del experimento, dejando sólo una planta por punto de siembra, previendo una población final de 62500 plantas por hectárea, aproximadamente.

Para determinar el efecto de la interacción genotipo ambiente y el potencial productivo en las variedades estudiadas, se utilizó el rendimiento de grano (RG), ajustado a $15 \%$ de humedad. Los ensayos fueron establecidos y cosechados en forma manual y el manejo agronómico se realizó conforme a las recomendaciones técnicas establecidas para el cultivo en cada ambiente de prueba.

El análisis estadístico fue realizado mediante un ANOVA individual y combinado para la media de cada unidad experimental, utilizando el procedimiento GLM del SAS.

Cuadro 1. Nombres y lugares de origen de las variedades de maíz amiláceo incluidas en el estudio

\begin{tabular}{|c|c|c|c|c|c|}
\hline Entrada & Ecotipo-Variedad & Tipo de variedad & $\begin{array}{l}\text { Código- } \\
\text { variedad }\end{array}$ & Procedencia & Distrito \\
\hline 1 & Astilla Amarilla-DH & Local & G1 & Los Ángeles de & \multirow{4}{*}{ Daniel Hernández } \\
\hline 2 & Astilla Blanca-DH & Local & $\mathrm{G} 2$ & Cucharán & \\
\hline 3 & Carhuay-DH & Local & G3 & Santa María & \\
\hline 4 & Carhuay-RDH & Local & G4 & Rundo & \\
\hline 5 & Carhuay-Q & Local & G5 & Anjara Pata & \multirow{3}{*}{ Quishuar } \\
\hline 6 & Bolón-Q & Local & G6 & Pachas & \\
\hline 7 & Chullpi-Q & Local & G7 & Yacutoclla & \\
\hline 8 & Astilla Blanca-GPP & Local & G8 & Gentil Pampas & \multirow{4}{*}{ Pampas } \\
\hline 9 & Astilla Blanca-ACP & Local & G9 & Ahuayta Casay & \\
\hline 10 & Cusqueado-P & Local & G10 & Gentil Pampas & \\
\hline 11 & Carhuay-P & Local & G11 & Mantacra & \\
\hline 12 & Astilla Blanca - Cusqueado & Local & G12 & \multirow[b]{2}{*}{$\begin{array}{l}\text { Agua dulce, } \\
\text { San Julián Alto }\end{array}$} & \multirow[b]{2}{*}{ Colcabamba } \\
\hline 13 & $\begin{array}{l}\text { Astilla Blanca - Astilla } \\
\text { Amarilla }\end{array}$ & Local & G13 & & \\
\hline 14 & Almidón-ST & Local & G14 & Buenos Aires & \multirow{4}{*}{$\begin{array}{l}\text { Santiago de } \\
\text { Tucuma }\end{array}$} \\
\hline 15 & Astilla Blanca-ST & Local & G15 & Huayrapire & \\
\hline 16 & Chullpi-Astilla Blanca & Local & G16 & Vuelo Pata & \\
\hline 17 & Chullpi-ST & Local & G17 & Usnupampa & \\
\hline 18 & Pistiada-Pi & Local & G18 & \multirow{2}{*}{ Muyupata } & \multirow{2}{*}{ Pichos } \\
\hline 19 & Carhuay-Pi & Local & G19 & & \\
\hline 20 & Choclero-101 & Testigo & $\mathrm{T} 1$ & \multirow{6}{*}{ INIA Cajamarca } & \multirow{6}{*}{ Cajamarca } \\
\hline 211 & INIA-603 Choclero & Testigo & $\mathrm{T} 2$ & & \\
\hline 221 & INIA-601 & Testigo & $\mathrm{T} 3$ & & \\
\hline 23 & Umutu & Testigo & $\mathrm{T} 4$ & & \\
\hline 24 & Chaucha Semi Blanco & Testigo & T5 & & \\
\hline 25 & Chaucha Rojo & Testigo & T6 & & \\
\hline
\end{tabular}


Cuadro 2. Localidades y fechas de siembra y cosecha de los experimentos

\begin{tabular}{|c|c|c|c|c|c|c|}
\hline \multirow{2}{*}{$\mathrm{N}^{\circ}$} & \multirow{2}{*}{$\begin{array}{c}\text { Nombre de } \\
\text { localidad }\end{array}$} & \multirow{2}{*}{ Localización } & \multicolumn{2}{|c|}{ Ubicación geográfica } & \multicolumn{2}{|c|}{ Fecha de siembra } \\
\hline & & & Latitud & Longitud & $\mathrm{msnm}$ & y de cosecha \\
\hline 1 & Beto Ega & $\begin{array}{l}\text { Finca Sr. Adalberto Ega, Anexo Ru } \\
\text { Distrito Daniel Hernández }\end{array}$ & $12^{\circ} 21^{\prime} 29^{\prime \prime} \mathrm{S}$ & $74^{\circ} 50^{\prime} 30^{\prime \prime} \mathrm{W}$ & 3217 & $\begin{array}{l}06 / 10 \\
27 / 06\end{array}$ \\
\hline 2 & UNAT & $\begin{array}{l}\text { Finca UNAT, Anexo Rundo, } \\
\text { Distrito Daniel Hernández }\end{array}$ & $12^{\circ} 21^{\prime} 17^{\prime \prime} \mathrm{S}$ & $4^{\circ} 50^{\prime} 10^{\prime \prime} \mathrm{W}$ & 3206 & $\begin{array}{l}23 / 10 / 2018 \\
26 / 06 / 2019\end{array}$ \\
\hline 3 & El Rosario & $\begin{array}{l}\text { Finca del Sr. Clímaco Culcapuza, } \\
\text { Centro Poblado Mantacra, Distrito Pam }\end{array}$ & $12^{\circ} 2$ & ' 43" W & 2860 & $\begin{array}{l}29 / 10 / 2018 \\
31 / 05 / 2019\end{array}$ \\
\hline 4 & Pilcos & $\begin{array}{l}\text { Finca de la Comunidad Pilcos, } \\
\text { Distrito, Colcabamba }\end{array}$ & $12^{\circ} 23^{\prime} 47^{\prime \prime} \mathrm{S}$ & $74^{\circ} 39^{\prime} 34^{\prime \prime} \mathrm{W}$ & 2483 & $\begin{array}{r}31 / 10 / 2018 \\
09 / 05 / 2019\end{array}$ \\
\hline 5 & Era Pata & $\begin{array}{l}\text { Finca de la Municipalidad } \\
\text { de Quishuar, Distrito Quishuar }\end{array}$ & $12^{\circ} 15^{\prime} 17^{\prime \prime} \mathrm{S}$ & $74^{\circ} 46^{\prime} 36^{\prime \prime} \mathrm{W}$ & 2914 & $\begin{array}{l}\text { 27/11/2018; } \\
07 / 08 / 2019\end{array}$ \\
\hline
\end{tabular}

El ANOVA combinado se efectuó conforme al modelo $Y_{i j k l}=\mu+t_{i}+R_{j(l)}+\beta_{k(j l)}+\lambda_{l}+t \lambda_{i l}+\varepsilon_{i j k l}$, donde $Y_{i j k l}$ representa la observación obtenida en el genotipo i, evaluado en la repetición $\mathrm{j}$, dentro de la localidad 1 , en el bloque $\mathrm{k} ; \mu$ es la media general; $t_{i}$ es el efecto fijo del genotipo; $R_{j(l)}$ es el efecto aleatorio de la repetición dentro de la localidad; $\beta_{k(j l)}$ es el efecto aleatorio del bloque dentro de la repetición y la localidad; $\lambda_{l}$ es el efecto aleatorio de la localidad; $\tau \lambda_{i l}$ es el efecto aleatorio de la interacción entre el genotipo y la localidad; $\mathcal{E}_{i j k l}$ es el error asociado a la observación $Y_{i j k l}$, conforme a Steel y Torrie (1988).Adicionalmente, se realizó una prueba de contraste ortogonal para comparar el potencial productivo de las variedades locales con el potencial expresado por las variedades mejoradas, utilizando el procedimiento GLM del SAS.

Una vez detectada la presencia de la interacción genotipo ambiente, se procedió a realizar el análisis multivariado, para obtener los valores singulares de los primeros términos AMMI significativos para los genotipos y ambientes, basados en los procedimientos establecidos por Vargas y Crossa (2000), utilizando sólo las medias ajustadas, por lo cual fue necesario proporcionar al programa la información correspondiente al valor del estimador del error combinado, los grados de libertad de dicho error y el número de repeticiones, de acuerdo al siguiente modelo matemático:

$$
Y_{i j}=\mu+g_{i}+a_{j}+\sum_{k=1}^{n} \lambda_{k} \gamma_{i k} \alpha_{j k}+\rho_{i j}+\varepsilon_{i j}
$$

donde $Y i j$ representa la media del genotipo $i$ en el ambiente $j ; \mu$ es la media general; $g_{i}$ y $a_{j}$ son los efectos del genotipo y el ambiente, respectivamente; $n$ es el número de componentes principales (CP) retenidos en el modelo; $k$ es el valor singular para cada $\mathrm{CP}$; $i k$ son valores de los vectores de los genotipos para cada $\mathrm{CP} ; j k$ son los valores de los vectores de los ambientes para cada $\mathrm{CP}$; $i j$ es el residual de la IGA; $\varepsilon_{i j}$ es el error experimental medio. Para cada genotipo y ambiente fueron obtenidos los valores genotípicos y ambientales por medio del procedimiento PROC IML del SAS. La significancia de cada $\mathrm{CP}$ fue medida mediante una prueba de $\mathrm{F}$ al nivel 0,05 de probabilidad, comparando el cuadrado medio de cada $\mathrm{CP}$, con el cuadrado medio del error experimental, conforme a Crossa et al. (1990). El número de ejes posibles (CP) que el modelo puede retener en AMMI es el mínimo (G-1; E-1); los ejes que resultaron no significativos se incluyeron en el residual y los valores del CP1 y el rendimiento medio de genotipos/ambientes fueron utilizados para construir el gráfico de doble representación biplot del modelo AMMI (García et al., 2009; García et al., 2020).

\section{RESULTADOS Y DISCUSIÓN}

El ANOVA combinado indicó que las variedades de maíz amiláceo no mantuvieron sus niveles de productividad en los ambientes de cultivo en los que se evaluaron, al mostrar diferencias estadísticas significativas $(P \leq 0,01)$ para el efecto de interacción genotipo por ambiente (Gen*Amb) (Cuadro 3), lo que sugiere que el desempeño productivo mostrado por las variedades, varió en función de la condición ambiental presente en cada localidad donde fueron cultivadas. Este análisis también reveló diferencias estadísticas significativas $(P \leq 0,01)$ entre los ambientes de cultivo y entre los genotipos, demostrando las diferencias en condiciones agroecológicas que caracterizaron a cada ambiente 
de evaluación y la divergencia genética entre las variedades utilizadas en el estudio.

Cuadro 3. Cuadrados medios para rendimiento de grano del ANOVA combinado para 25 variedades de maíz amiláceo evaluadas en cinco ambientes de la provincia de Tayacaja en el ciclo de cultivo 2018-2019

\begin{tabular}{lcc}
\hline Fuente de variación & GL & $\begin{array}{c}\text { Rendimiento } \\
\text { de grano }\end{array}$ \\
\hline Ambientes (Amb) & 4 & $215,75^{* *}$ \\
Repeticiones/(Amb) & 10 & 0,73 \\
Bloques/(Amb*Rep) & 60 & $0,88^{*}$ \\
Genotipos (Gen) & 24 & $2,83^{*}$ \\
Gen*Amb & 96 & $1,86^{* *}$ \\
Covarianza & 1 & $3,36^{*}$ \\
Error & 179 & 0,59 \\
Total & 374 & \\
\hline & $\mathrm{CV}(\%)$ & 17,76 \\
\hline
\end{tabular}

$\mathrm{GL}=$ grados de libertad $\mathrm{CV}=$ Coeficiente de variación. $*$ $\mathrm{y} * *$ indican diferencias significativas al $5 \%$ y $1 \%$, respectivamente

Falcon et al. (2020) evaluaron la IGA utilizando diferentes caracteres de planta, incluyendo los componentes del rendimiento, y encontraron que la IGA explicó entre 9.0 y $20.4 \%$ de la varianza fenotípica, con mayores efectos para los componentes del rendimiento. En este estudio, la IGA explicó alrededor del $14 \%$ de la variación fenotípica del rendimiento de las variedades de maíz amiláceo.

A pesar de que la localidad Pilcos, Colcabamba, no mostró homogeneidad de varianza para el error experimental, el análisis combinado se hizo incluyendo este ambiente de prueba, puesto que no hubo diferencia en los resultados cuando se excluyó esta localidad del ANOVA combinado.

Por su parte, el análisis de varianza individual por localidad corroboró diferencias estadísticas significativas $(P \leq 0,01)$ para el efecto de variedades en todos los ambientes de cultivo, a excepción de la localidad de Quishuar, en donde estadísticamente, todas las variedades demostraron el mismo potencial productivo (Cuadro 4), resultado que permitió aseverar una elevada diversidad genética entre los genotipos estudiados. Asimismo, el análisis individual demostró que fue importante considerar el número de plantas por parcela como covariable del rendimiento de grano, al observarse efectos de covarianza en dos de los cinco ambientes de evaluación (Cuadro 4). Los coeficientes de variación se ubicaron entre 12,30 a 19,43, valores considerados apropiados para este tipo de variables.

Cuadro 4. Cuadrados medios para rendimiento de grano según el análisis individual por localidad, para 25 variedades de maíz amiláceo evaluadas en cinco localidades de la provincia de Tayacaja en el ciclo de cultivo 2018-2019

\begin{tabular}{lcclllc}
\hline \multicolumn{1}{c}{ Fuente de Variación } & GL & Beto Ega & UNAT & Mantacra & Pilcos & Quishuar \\
\hline Repeticiones (Rep) & 2 & 0,077 & 0,933 & 1,990 & $0,205^{*}$ & 0,526 \\
Bloques/(Rep) & 12 & $0,739^{*}$ & $0,919^{* *}$ & 1,907 & 0,033 & 1,065 \\
Genotipos (Gen) & 24 & $1,479^{* *}$ & $2,168^{* *}$ & $5,078^{* *}$ & $0,824^{* *}$ & 0,512 \\
Covarianza & 1 & 0,081 & $3,340^{* *}$ & 3,225 & $0,669^{* *}$ & 0,041 \\
Error & 35 & 0,286 & 0,302 & 1,303 & 0,036 & 0,994 \\
Total & 74 & & & & & \\
\hline \multicolumn{2}{c}{ Media $\left(\mathrm{Mg}^{2}\right.$ ha $\left.^{-1}\right)$} & 3,524 & 4,137 & 6,995 & 2,018 & 5,013 \\
\hline
\end{tabular}

$\overline{\mathrm{GL}}=$ grados de libertad; $\mathrm{CV}=$ Coeficiente de variación; * $\mathrm{y} * *$ indican diferencias significativas al $5 \% \mathrm{y}$ $1 \%$, respectivamente

Como se observa en el Cuadro 4, Mantacra resultó ser el ambiente más favorable, al presentar un potencial productivo de cerca de $7 \mathrm{Mg} \cdot \mathrm{ha}^{-1}$, mientras que la localidad de Pilcos resultó con el promedio más bajo de productividad (cerca de 2
$\left.\mathrm{Mg} \cdot \mathrm{ha}^{-1}\right)$. Es importante indicar que la localidad de Pilcos, fue afectada por un ataque de aves que se presentó en las etapas R3-R4 (grano lechoso a pastoso) del cultivo, perjudicando significativamente la producción en todas las variedades 
estudiadas; el análisis preliminar de estos daños indicó una reducción promedio del $50 \%$ en rendimiento, en donde las variedades INIA-601, Chulpy, Umutu y Choclero-101 fueron las más afectadas por las aves (García y Prieto, 2019).

Los resultados de producción (Cuadro 5) indicaron que las variedades experimentales Astilla Blanca-Cusqueado (G12), Cusqueado-P (G10) y Astilla Blanca-DH (G2), fueron las que presentaron los mayores niveles de productividad promedio, superando significativamente $(P \leq 0,05)$ a la mayoría del resto de variedades experimentales y al testigo Chaucha Rojo (T6); no así al resto de las variedades utilizadas como testigos, las cuales resultaron con niveles de productividad promedio similares (T2 y T4) o incluso superior (T1) al rendimiento medio observado en la variedad experimental Astilla Blanca-DH (G2).

Cuadro 5. Medias de rendimiento (ordenadas en forma descendente) y componentes principales (CP1, CP2 y CP3) en la evaluación de la IGA de 25 variedades de maíz amiláceo (ciclo de cultivo 20182019)

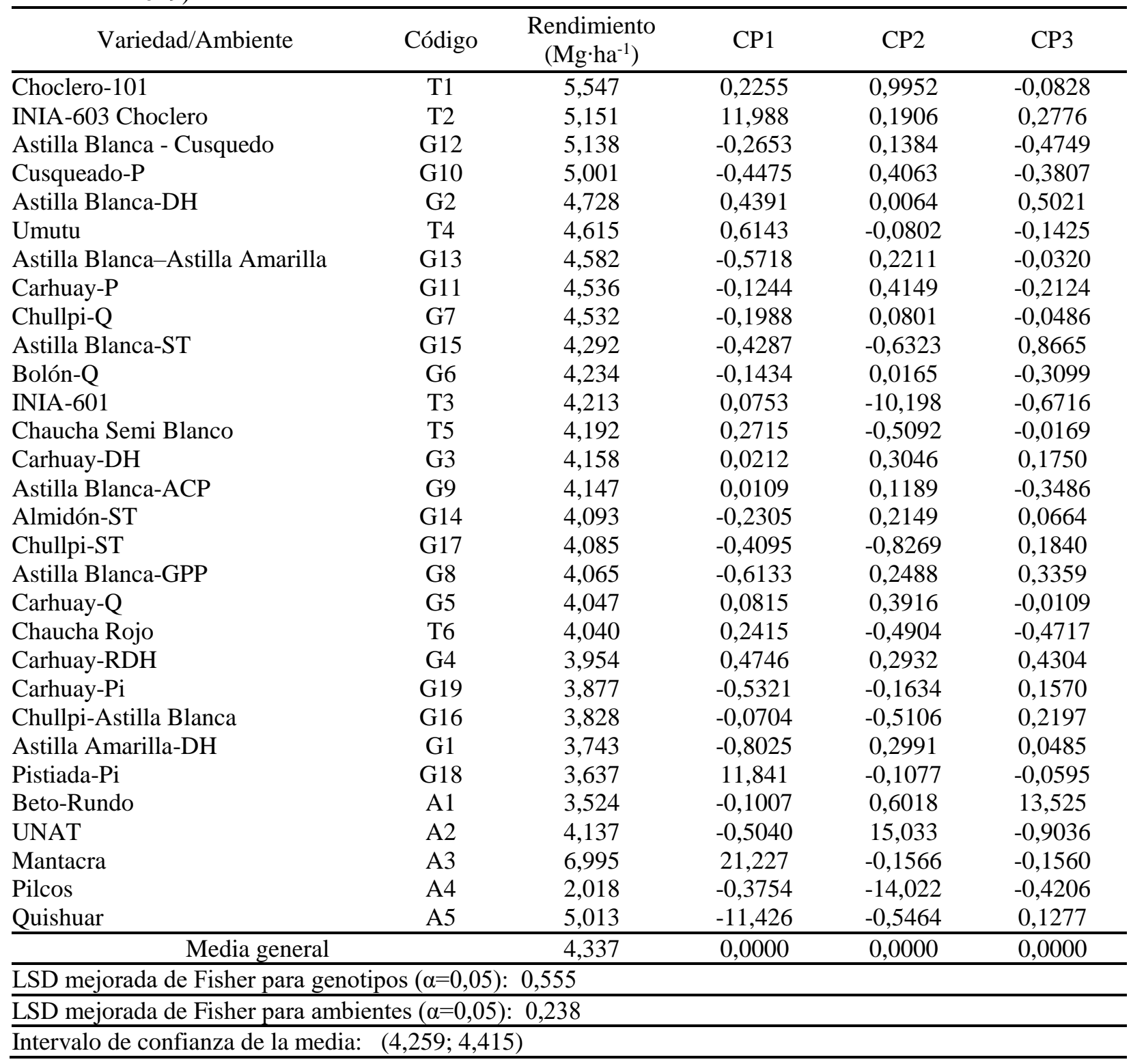

Las variedades Choclero-101 (T1) e INIA-603 Choclero (T2) mostraron el mayor potencial de productividad promedio, con 5,547 y 5,151 $\mathrm{Mg} \cdot \mathrm{ha}^{-1}$, respectivamente, superando de manera 
significativa $(P \leq 0,05)$ al resto de las variedades incluidas en el estudio, excepto a las variedades Cusqueado-P $\left(\mathrm{G}_{10}\right)$ con 5,001 Mg.ha- ${ }^{-1}$ y Astilla Blanca-Cusqueado $\left(\mathrm{G}_{12}\right)$ con $5,138 \mathrm{Mg} \cdot \mathrm{ha}^{-1}$, que mostraron niveles de productividad promedio estadísticamente iguales $(P>0,05)$ a la variedad testigo $\mathrm{T}_{2}$.

El contraste ortogonal realizado para comparar el conjunto de variedades experimentales con las mejores variedades testigo demostró la superioridad en rendimiento de los testigos $\mathrm{T}_{1}$ y $\mathrm{T}_{2}$; mientras que el mismo análisis realizado para comparar la media del conjunto de variedades experimentales contra la media del conjunto de variedades testigo, reveló diferencias estadísticas significativas $(P \leq 0,01)$ entre ambas medias (Cuadro 6), indicando la superioridad en productividad de las variedades mejoradas.

Cuadro 6. Cuadrados medios para rendimiento de grano de los contrastes ortogonales realizados entre las variedades locales vs las variedades testigos, según el análisis combinado de 25 variedades de maíz amiláceo evaluadas en cinco localidades de la provincia de Tayacaja en el ciclo de cultivo $2018-2019$.

\begin{tabular}{lccc}
\hline \multicolumn{1}{c}{ Contraste ortogonal } & \multicolumn{2}{c}{ GL Cuadrados medios } \\
\hline Locales vs Choclero-101 & 1 & $19.393 * *$ \\
Locales vs INIA-603 Choclero & 1 & $9.343^{* *}$ \\
Locales vs INIA-601 & 1 & 0.012 \\
Locales vs Testigos (INIA) & 1 & $6.865 * *$ \\
\hline GL = grados de libertad. & $* *$ indica diferencias \\
significativas al 1\% para el contraste de las medias
\end{tabular}

La Figura 1 muestra estos resultados, que demuestran la importancia del mejoramiento genético para desarrollar genotipos de superior desempeño agronómico y adaptado a las condiciones agroecológicas de las regiones de producción comercial. Al respecto, Carena (2005) señala que los esfuerzos para el desarrollo de poblaciones élites han demostrado que el mejoramiento de germoplasma es altamente valioso y merece la financiación pública, esfuerzos que deben ser apoyados para permitir el desarrollo de diversas fuentes de líneas endogámicas élite y el desarrollo de híbridos a partir de poblaciones mejoradas para mercados específicos (por ejemplo, orgánicos), que permitan diversificar las opciones de los productores. La Figura 1 muestra que el rendimiento medio de la variedad INIA-601 fue estadísticamente igual $(P>0,05)$ a la media del conjunto de variedades locales incluidas en el estudio; no obstante, esta variedad, distinguida por su elevado contenido de vitaminas, minerales esenciales y antocianinas, es la que mayores beneficios aporta a la salud humana (Lao et al., 2017; Khamphasan et al., 2018). En consecuencia, INIA-601 puede proporcionar una mayor rentabilidad al productor frente a la que podrían las variedades más productivas, pero carentes de este valor adicional.

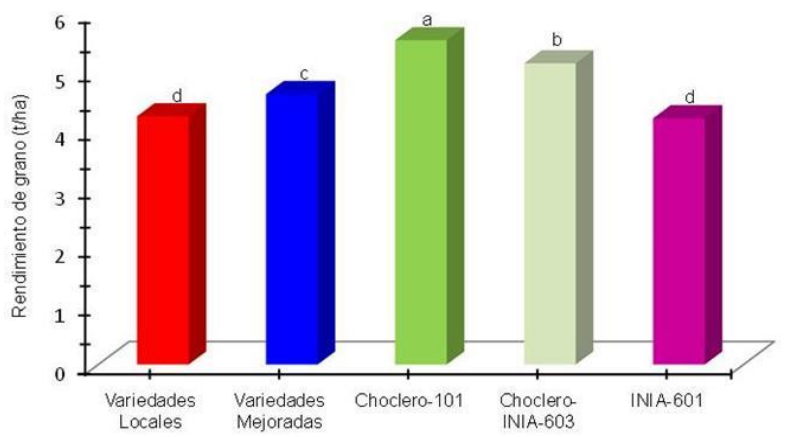

Figura 1. Comparación de los niveles medios de productividad de variedades locales y mejoradas. Letras diferentes sobre las barras indican diferencias significativas $(P \leq 0,05)$ entre las medias, según el análisis de contrastes

Análisis AMMI. Los resultados del análisis AMMI, indicaron que los tres primeros ejes fueron estadísticamente significativos $(P \leq 0,01$, para $\mathrm{CP} 1$ y $\mathrm{CP} 2$ y $P \leq 0,05$, para $\mathrm{CP} 3$ ), explicando alrededor del $96 \%$ de la interacción genotipo ambiente; el CP1 explicó un poco más del $52 \%$ de la IGA, en tanto que entre el CP1 y el $\mathrm{CP} 2$ explicaron alrededor del $85 \%$ de la variación debida a la IGA (Cuadro 7). Estos resultados mostraron que una elevada proporción de la variación correspondiente a la IGA fue explicada por el modelo AMMI-1 con el $78 \%$ de los grados de libertad de la interacción, generando un residual que apenas representó cerca del $4 \%$ del efecto de IGA, siendo éste estadísticamente no significativo $(P>0,05)$, lo que permitió inferir que el modelo fue apropiado para explicar la interacción genotipo ambiente en el conjunto de genotipos estudiados.

García et al. (2009) llegaron a conclusiones similares al utilizar el modelo AMMI para evaluar la estabilidad del rendimiento y el potencial agronómico de nueve cultivares de maíz en ocho 
localidades de Venezuela. De la misma forma, Crossa et al. (1990), San Vicente et al. (2005) y García et al. (2020) realizaron una interpretación similar de los resultados del ANOVA del modelo AMMI. No obstante, en lo que respecta a la proporción de la IGA que fue explicada por el CP1, los resultados difieren de lo observado en estudios previos realizados en diferentes ambientes tropicales, los cuales, utilizando el modelo AMMI para evaluar la IGA y la estabilidad del rendimiento de diferentes genotipos de maíz, incluyendo híbridos y variedades de libre polinización, encontraron que el CP1, por sí sólo, explicó una proporción superior al 60 \% de la IGA (García et al., 2009; González et al., 2009). Difieren también de lo observado en otros estudios, en donde los dos primeros $\mathrm{CP}$ apenas lograron explicar valores cercanos al $60 \%$ de la variación debida a la IGA (San Vicente et al., 2005; Lozano et al., 2015). De igual manera, Balestre et al. (2009) encontraron que los dos primeros CP capturaron sólo el 42,77 \% de la variación debida a la IGA al utilizar el modelo AMMI para evaluar la estabilidad y adaptabilidad de 45 híbridos dobles de maíz en 15 localidades de Brasil. Similarmente, Falcon et al. (2020) utilizaron el modelo AMMI para examinar la IGA en 31 líneas endocriadas de maíz en 36 ambientes contrastantes de Estados Unidos y encontraron que entre el $\mathrm{CP} 1$ y el $\mathrm{CP} 2$ representaron entre 32,5 y $52,4 \%$ de la variación observada para cada carácter estudiado.

Cuadro 7. Cuadrados medios del análisis de varianza combinado con efectos lineales y multiplicativos del modelo AMMI, para el rendimiento de grano de 25 variedades de maíz amiláceo evaluadas en 5 ambientes de la provincia de Tayacaja, en el ciclo de cultivo 2018-2019

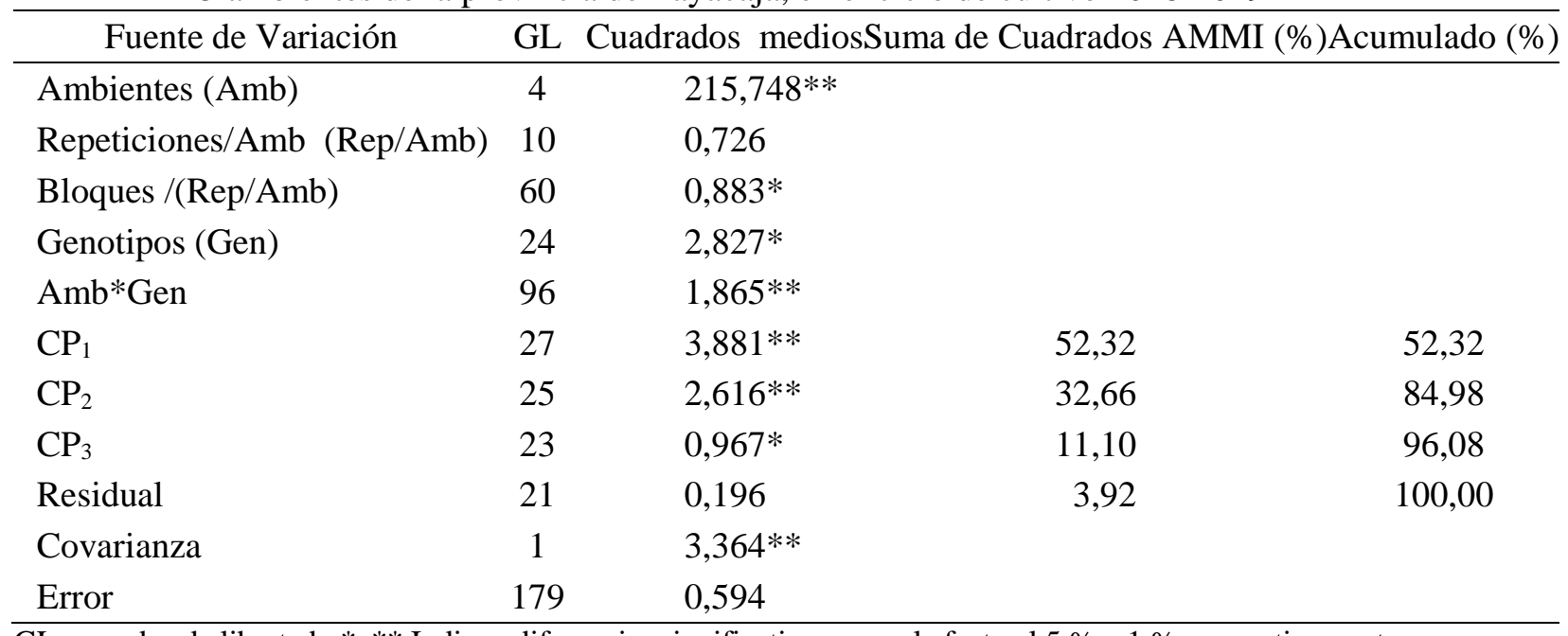

$\mathrm{GL}=$ grados de libertad. $*$, ** Indican diferencias significativas para el efecto al $5 \%$ y $1 \%$, respectivamente

Por su parte, Vieira et al. (2009) utilizaron dos métodos multivariados, incluyendo el AMMI para evaluar la IGA en nueve cultivares de maíz reventón o canchita ( los dos primeros CP explicaron un porcentaje de la variación debida a la IGA similar al observado en este trabajo, con valores de alrededor del $85 \%$. En el caso del trabajo de Ngaboyisonga et al. (2016), en el que se utilizó el método AMMI para evaluar la IGA de 18 híbridos más cinco variedades de libre polinización (OPVs), establecidos en nueve ambientes de mediana altitud y seis de elevada altitud en Ruanda, África, se obtuvieron diferentes resultados dependiendo de la condición ambiental; en los ambientes de mediana altitud, los dos primeros ejes sólo explicaron 54,65 \% de la variación total debida a la IGA, mientras que en los ambientes de elevada altitud los dos primeros CP explicaron $80,20 \%$ de la IGA.

La falta de correspondencia entre los resultados encontrados por San Vicente et al. (2005) respecto a lo observado en trabajos previos, fue atribuida a diversos factores relacionados con la complejidad del carácter y a la diversidad de genotipos, además de las condiciones de ambientes incluidos en los estudios. Efectivamente, en atributos como el rendimiento, caracterizado por estar determinado por un gran número de genes, cada uno de éstos con un efecto muy pequeño sobre el carácter y además fuertemente afectados por las condiciones de 
ambientes en donde se desarrollan los genotipos (Hallauer et al., 2010), en lo que respecta a los efectos de la IGA, difícilmente se pueden tener condiciones para que dos estudios puedan generar resultados similares. De allí la necesidad de utilizar estas herramientas de la estadística multivariada, que facilitan la selección de los genotipos con adaptación específica a cada condición de ambiente particular o de amplia adaptación general.

En el Perú se han llevado a cabo pocos trabajos para evaluar el efecto de interacción genotipo ambiente utilizando métodos multivariados. López et al. (2019) utilizaron el modelo AMMI para determinar la estabilidad y la IGA del rendimiento de 25 híbridos de maíz amarillo evaluados en cinco ambientes del país, y reportaron una variación debida a la IGA de 70,47 \% determinada por los dos primeros ejes del modelo. Por su parte, Chura y Huanuqueño (2015) reportaron los resultados de un estudio efectuado para evaluar el comportamiento de 20 genotipos de maíz amarillo, evaluados en cuatro ambientes de la costa peruana, pero no pudieron determinar los mejores genotipos para todos los ambientes utilizados o con adaptación específica a condiciones de ambientes particulares, debido a que sólo utilizaron el análisis univariado.

De acuerdo a los resultados del modelo AMMI, las variedades locales Astilla Blanca-Cusqueado (G12), Carhuay-P $\left(\mathrm{G}_{11}\right)$ y Chullpi-Q $\left(\mathrm{G}_{7}\right)$ resultaron con niveles de productividad promedio estadísticamente superiores $(P \leq 0,05)$ a la media general y con elevada consistencia a través de los ambientes (Figura 2). Del mismo modo, las variedades locales Astilla Blanca-GPP $\left(\mathrm{G}_{8}\right)$, Carhuay-DH $\left(\mathrm{G}_{3}\right)$, Astilla Blanca-ACP $\left(\mathrm{G}_{9}\right)$, Carhuay-Q $\left(\mathrm{G}_{5}\right)$, Almidón-ST $\left(\mathrm{G}_{14}\right)$ y ChullpiAstilla Blanca $\left(\mathrm{G}_{16}\right)$ presentaron un desempeño muy estable en los ambientes de evaluación, pero con niveles de productividad por debajo a la media general de los ensayos. Asimismo, el análisis AMMI demostró que la variedad Choclero-101 $\left(\mathrm{T}_{1}\right)$, además de ser el material con mayor nivel de productividad, tuvo un desempeño muy estable a través de los ambientes. Las variedades testigos INIA-601 $\left(\mathrm{T}_{3}\right)$, Chaucha Semi Blanco $\left(\mathrm{T}_{5}\right) \mathrm{y}$ Chaucha Rojo $\left(\mathrm{T}_{6}\right)$ resultaron con niveles de productividad muy estables a través de los ambientes, pero con rendimientos estadísticamente iguales $(P>0,05)$ para $\left(\mathrm{T}_{3}\right.$ y $\left.\mathrm{T}_{5}\right)$ o inferiores $\left(\mathrm{T}_{6}\right)$ a la media general de todos los experimentos. La variedad Choclero-INIA-603 $\left(\mathrm{T}_{2}\right)$ ocupó el segundo nivel más alto de productividad promedio, mostrando su mejor desempeño en la localidad de Mantacra $\left(\mathrm{A}_{3}\right)$.

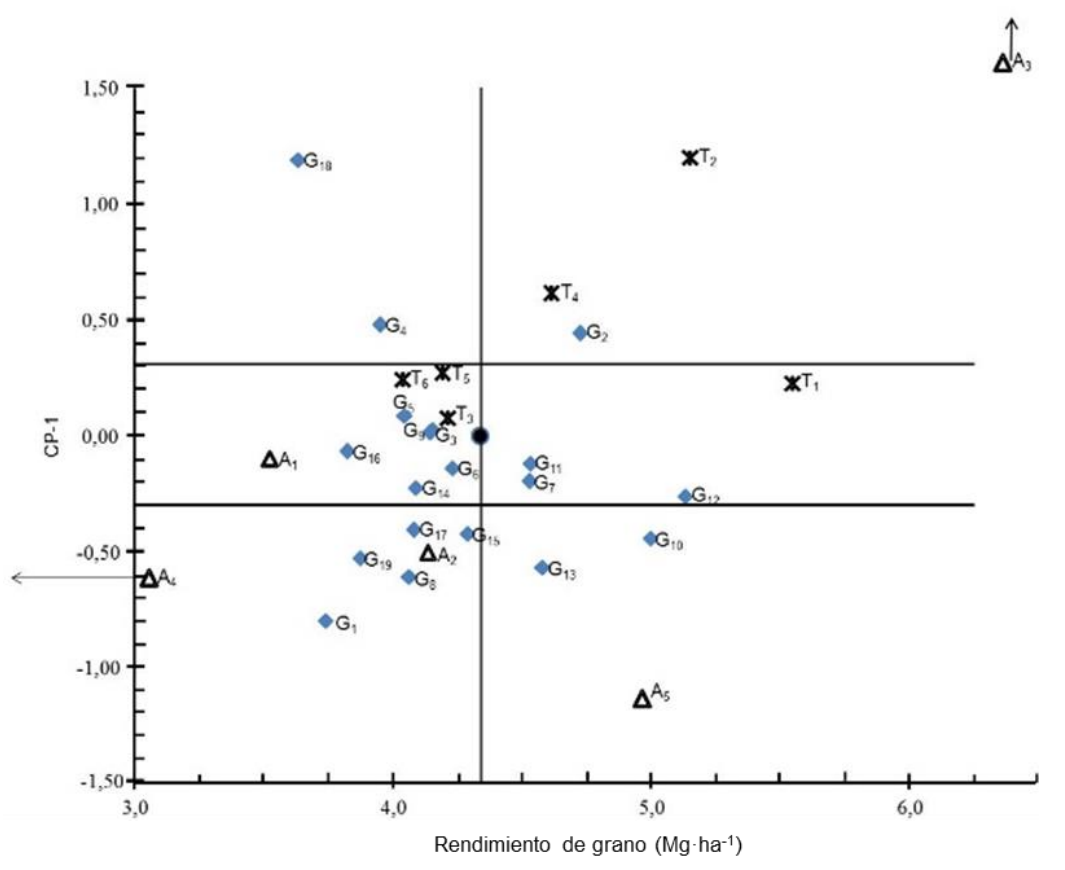

Figura 2. Modelo AMMI del comportamiento de 25 variedades ( $\mathrm{G}$ y $\mathrm{T}$ ) de maíz amiláceo evaluado en cinco ambientes (A) de la provincia de Tayacaja, Perú (ciclo de cultivo 2018-2019). Las flechas en $\mathrm{A}_{3}$ y $\mathrm{A}_{4}$ indican que esas localidades quedaron ubicadas fuera del gráfico 
En trabajos previos, para estimar el desempeño de diversos cultivares de maíz y en varios ambientes tropicales, utilizando el modelo AMMI, se han encontrado resultados similares, al identificar cultivares cuyos rendimientos resultaron consistentes a través de los ambientes de prueba, pero con niveles de productividad promedio por debajo de la media general (San Vicente et al., 2005; García et al., 2009; Andrés et al., 2014). Este tipo de estabilidad clasificada como biológica por Becker (1981), resulta poco útil en la agricultura, dado que los productores demandan cultivares que muestren comportamiento consistente a través de los ambientes, pero que al mismo tiempo respondan favorablemente a las mejoras ambientales, lo que significa, cultivares que presenten el mejor desempeño bajo cualquier condición ambiental, es decir, con estabilidad agronómica (García et al., 2009).

Los resultados del análisis del modelo AMMI también indicaron que la localidad Beto $\left(\mathrm{A}_{1}\right)$ fue el ambiente que menos contribuyó con la IGA, pudiendo considerarse como un ambiente neutral, con poca capacidad para discriminar entre los genotipos probados, mientras que el resto de los ambientes y particularmente las localidades Mantacra $\left(\mathrm{A}_{3}\right)$ y Quishuar $\left(\mathrm{A}_{5}\right)$, resultaron con mejores condiciones para discriminar a los genotipos evaluados, pudiéndose sugerir como ambientes claves para futuros estudios.

Las variedades $T_{1}, G_{18}, G_{1}, G_{8}$ y $G_{13}$ fueron las que más contribuyeron con la IGA, al mostrar los valores de CP1 más extremos, mientras que las variedades $T_{3}, G_{3}, G_{9}$ y $G_{5}$ fueron las que menos aportaron al efecto de la IGA, con valores de CP1 muy cercanos a cero (Figura 2). Estudios previos en diversos ambientes tropicales del mundo, señalan la utilidad del modelo AMMI para la identificación de los genotipos de superior comportamiento, con amplia adaptación o adaptación específica a los ambientes de prueba (Lunezzo et al., 2010; Chimonyo et al., 2014; Ngaboyisonga et al., 2016). En este estudio, los resultados sugieren que el modelo AMMI fue apropiado para evaluar la IGA y para determinar la capacidad discriminatoria de los ambientes de prueba.

Indudablemente, la interacción genotipo por ambiente representa un fenómeno biológico muy complejo, en donde el efecto de años y localidades (ambientes) y de los genotipos incluidos en los estudios, determinan la magnitud y grado de complejidad de dicho efecto; es en estos casos que las metodologías multivariadas facilitan la interpretación del comportamiento de los genotipos a través de los ambientes de evaluación, ayudando a los mejoradores y agrónomos en la selección de los genotipos más estables y de los mejores ambientes para llevar a cabo las evaluaciones. Al respecto, Yan et al. (2001) y Yan et al. (2007) refieren que en estudios para la determinación de mega-ambientes, se debe considerar evaluar diversas localidades durante varios años de prueba, para establecer los grupos de ambientes que consistentemente definen los mejores cultivares $o$ genotipos. Asimismo, Yan et al. (2007) señalan que para poder llegar a conclusiones definitivas, un mismo grupo de genotipos debe ser evaluado en un mismo grupo de ambientes durante varios años.

\section{CONCLUSIONES}

La IGA resultó altamente significativa y explicó alrededor del $14 \%$ de la variación fenotípica del rendimiento de las variedades de maíz amiláceo, en donde, de acuerdo al modelo utilizado, las variedades T1, G18, G1, G8 y G13 fueron las que más contribuyeron con este efecto, mientras que T3, G3, G9 y G5 resultaron genotipos neutrales, con poca contribución al fenómeno.

Los cultivares Astilla Blanca-DH (G2), ChullpiQ (G7), Cusqueado-P (G10), Carhuay-P (G11), Astilla Blanca-Cusqueado (G12), y Astilla BlancaAstilla Amarilla (G13) fueron las variedades locales que mostraron el mejor potencial productivo, pero sólo G7, G11 y G12 fueron estables a través de los ambientes de evaluación.

La variedad Choclero-101, utilizada como testigo, mostró el mayor potencial productivo y alta estabilidad para los ambientes evaluados, mientras que la variedad Choclero-INIA-603 (T2) ocupó el segundo nivel más alto de productividad promedio, pero su mejor desempeño lo mostró en la localidad de Mantacra.

\section{AGRADECIMIENTO}

Al Fondo de Desarrollo Socioeconómico de Camisea (FOCAM) por el financiamiento suministrado para llevar a cabo esta investigación y a los productores agrícolas Adalberto Ega y Clímaco Culcapusa Ilizarbe, a la Municipalidad de 
Quishuar y a la Comunidad Campesina Pilcos, por el apoyo recibido para llevar a cabo en sus predios algunos de los experimentos.

\section{LITERATURA CITADA}

1. Acevedo Barona, M., R. Álvarez, R. Silva, O. Torres y E. Reyes. 2019. Interacción genotipo ambiente en arroz para identificar megaambientes y ambientes ideales mediante el modelo de regresión por sitios y biplot. Bioagro 31(1): 35-44.

2. Admassu, S., M. Nigussie y H. Zelleke. 2008. Genotype-environment interaction and stability analysis for grain yield of maize (Zea mays L.) in Ethiopia. Asian Journal of Plant Sciences 7: 163-169.

3. Andrés-Meza, P., M. Sierra-Macías, J. MejíaContreras, J. Molina-Galán, A. EspinosaCalderón, N. Gómez-Montiel y R. ValdiviaBernal. 2014. Genotype-environment interaction in tropical maize varieties developed for the tropical region of Veracruz, México. Interciencia 39(3): 180-184.

4. Balestre, M., R. Von Pinho, J. Souza y R. Oliveira. 2009. Genotypic stability and adaptability in tropical maize based on AMMI and GGE biplot analysis. Genet. Mol. Res. 8(4): 1311-1322.

5. Becker, H. C. 1981. Correlations among some statistical measures of phenotypic stability. Euphytica 30: 835-840.

6. Carena, M. J. 2005. Maize commercial hybrids compared to improved population hybrids for grain yield and agronomic performance. Euphytica 141: 201-208.

7. Chimonyo, V., C. Mutengwa y C. Chiduza. 2014. Genotype x environment interactions and yield stability of stress-tolerant openpollinated maize varieties in the Eastern Cape province, South Africa. South African Journal of Plant and Soil 31(2): 61-68.

8. Chura C. y E. Huanuqueño C. 2015. Comportamiento de ocho poblaciones de maíz amarillo (Zea mays L.) en cruzas con un probador. Anales Científicos 76(1): 78-86.

9. Crossa, J., H. Gauch y R. Zobel. 1990. Additive main effects and multiplicative interaction analysis of two international maize cultivar trials. Crop Sci. 30: 493-500.

10.Crossa, J., P. Cornelius y M. Vargas. 2000. Modelos Estadísticos Multiplicativos para el Análisis de la lnteracción Genotipo x Ambiente. CIMMYT Library. México D. F., México. https://repository.cimmyt.org/bitstre am/handle/10883/3487/68592.pdf?sequence= $1 \&$ isAllowed=y (consulta de junio 18, 2020).

11.Dudley, J.W. 1997. Quantitative Genetics and Plant Breeding. Advances in Agronomy 59: 123.

12.Falcon, C., S. Kaeppler, E. Spalding, N. Miller, N. Haase, N. AlKhalifah et al. 2020. Relative utility of agronomic, phenological, and morphological traits for assessing genotype-by-environment interaction in maize inbreds. Crop Science 60(1): 62-81.

13.García, P., S. Cabrera, A. Pérez, R. Silva, R. Álvarez, C. Marín et al. 2009. Estabilidad del rendimiento y potencial agronómico de cultivares de maíz de endospermo normal y QPM en zonas agroecológicas de Venezuela. Agronomía Tropical 59(4): 433-443.

14.García M., P.J., A.A. Pérez C., R.J. Silva D., R.M. Álvarez P., P. Monasterio P. y L.A. Taramona R. 2020. Evaluación del potencial agronómico de híbridos de maíz amarillo basado en el análisis GGE Biplot y el modelo AMMI. Bioagro 32(2): 95-106.

15.Gauch, H.G. y R.W. Zobel. 1996. AMMI analysis of yield trials. In: M.S. Kang y H. G. Gauch. (eds.). Genotype-by-Environment interaction. CRC Press. Boca Raton, FL, USA. pp. 85-122.

16.González, A. J. Sahagún, L. Vázquez, J. Rodríguez, D. Pérez, A. Domínguez, O. Franco, y A. Balbuena. 2009. Identificación de variedades de maíz sobresalientes considerando el modelo AMMI y los Índices de Eskridge. Agricultura Técnica en México 35(2): 189-200.

17. Hallauer, A., M. Carena y J. Miranda Filho. 2010. Quantitative Genetics in Maize Breeding. Springer. London.

18. Khamphasan, P., K. Lomthaisong, B. Harakotr, D. Ketthaisong, M. Scott, K. Lertrat y B. Suriharn. 2018. Genotypic variation in anthocyanins, phenolic compounds, and antioxidant activity in cob and husk of purple 
field corn. Agronomy 8: 271: 1-15.

19.Lao, F., G. Sigurdson, y M. Giusti. 2017. Health benefits of purple corn (Zea mays L.) phenolic compounds. Comprehensive Reviews in Food Science and Food Safety 16: 234-246.

20.López-Morales, F., J. Chura-Chuquija y G. García-Pando. 2019. Interacción genotipo por ambiente del rendimiento de maíz amarillo en híbridos trilineales, Perú. Revista Mexicana de Ciencias Agrícolas 10(4): 859-872.

21.Lozano-Ramírez, A., A. Santacruz-Varela, F. San-Vicente-García, J. Crossa, J. Burgueño y J. Molina-Galán. 2015. Modelación de la interacción genotipo $\mathrm{x}$ ambiente en rendimiento de híbridos de maíz blanco en ambientes múltiples. Rev. Fitotec. Mex. 38(4): 337-347.

22.Lunezzo R., V. García, M. Balestre y D. Ferreira. 2010. Evaluation of maize hybrids and environmental stratification by the methods AMMI and GGE biplot. Crop Breeding and Applied Biotechnology 10: 247253.

23.Mudada N., J. Chitamba, T. Macheke, y P. Manjeru. 2017. Genotype x environmental interaction on seed cotton yield and yield components. Open Access Library Journal 4: $1-22$.

24.Ngaboyisonga, C., A. Nyombayire, M. Gafishi, F. Nizeyimana, A. Uwera, T. Ndayishimiye et al. 2016. Adaptability and genotype by environment interaction of maize commercial hybrid varieties from East African seed companies in Rwandan environments. Global Journal of Agricultural Research 4(2): 32-40.

25.San Vicente, F., C. Marín y D. Díaz. 2005. Estabilidad del rendimiento y potencial agronómico de híbridos de maíz de alta calidad de proteína (QPM) en Venezuela.
Agronomía Tropical 55(3): 397-410.

26.Souza, A., G. Miranda, M. Gonzaga y L. Souza. 2009. Predicting the genetic gain in the Brazilian white maize landrace. Ciência Rural 39: 19-24.

27.Steel, R. y J. Torrie 1988. Bioestadística: Principios y Procedimientos. McGraw-Hill /Interamericana, México.

28.UNAT (Universidad Nacional Autónoma de Tayacaja "Daniel Hernández Morillo"). 2018. Selección de cultivares y conservación de la identidad genética de variedades de maíz (Zea mays L.) en la provincia de Tayacaja. Proyecto aprobado según Resolución de la Comisión Organizadora $\mathrm{N}^{\circ}$ 0126-2018-CO-UNAT. 49 p. https://unat.edu.pe/nuestras-investigaciones/ (consulta de junio 18, 2020).

29. Vargas H., M. y J. Crossa. 2000. El análisis AMMI y la gráfica del biplot en SAS. Universidad Autónoma de Chapingo (UACh) y Unidad de Biometría y Estadística, CIMMYT. México, DF. 42 p. https://reposito ry.cimmyt.org/bitstream/handle/10883/3489/ 73248.pdf? sequence $=1 \&$ is Allowed $=y$ (consulta de noviembre 1, 2016).

30.Vieira, G., L. Vagno, G. Moreira, H. Namorato, L. Rodrigues, y M. Oliveira. 2009. Multivariate analyses of genotype $\mathrm{x}$ environment interaction of popcorn. Pesq. Agropec. Bras. 44(1): 45-50.

31.Yan, W., M. Kang, B. Ma, S. Woods y P. Cornelius. 2007. GGE Biplot vs. AMMI Analysis of Genotype-by-Environment Data. Crop Sci. 47: 641-653.

32. Yan, W., P. Cornelius, J. Crossa y L. Hunt. 2001. Two types of GGE biplots for analyzing multi-environment trial data. Crop Sci. 41: 656-663.

33.Zobel, R.W., M.J. Wright y H.G. Gauch. 1988. Statistical analysis of a yield trial. Agron. J. 80: 388-393. 